\title{
The acoustic resonance of rectangular and cylindrical cavities
}

\author{
A. Rona* \\ Department of Engineering, University of Leicester, Leicester LE1 7RH, UK
}

\begin{abstract}
An investigation is conducted on the acoustic resonance that develops in rectangular and cylindrical cavities with rigid walls. An analytical model for the small amplitude acoustic perturbations inside an enclosure with rigid walls is developed from classical linerized acoustics. The method is first applied to a rectangular geometry and the normalized mode shapes and frequencies of the first six standing wave modes are given. The results are used to diagnose whether coupling is likely to occur between the first two Rossiter modes and the acoustic standing waves, which may lead to a reinforcement of the flow instability. At the selected test conditions, the method indicates the likelyhood of such coupling for the second Rossiter mode. The acoustic resonant mode predictions for a cylindrical cavity of length to depth ratio 0.71 and 2.5 , tested at near-incompressible speeds, suggest that the acoustic resonant modes and the fluid dynamic instability in the enclosure are sufficiently apart in frequency not to strongly interact with one another. The parametrized analytical solutions developed in this study enable the aeroacoustic engineer to diagnose whether coupling between a given fluid dynamic instability and acoustic resonance is likely to affect a rectangular or cylindrical cavity component.
\end{abstract}

\section{Introduction}

$\mathrm{T}^{\mathrm{w}, \mathrm{g}}$ HE grazing flow past a surface cut-out can develop instabilities that adversely affect the aerodynamic 1 performance of airframe, automotive and railway components. Such instabilities can be either fluid dynamic, hydroelastic or flow-resonant, depending on whether aeroelastic or acoustic effects contribute to the flow unsteadiness.

The flow past the surface cut-out, or cavity, separates at the upstream edge, forming a shear layer, as shown in figure 1. Provided the cavity streamwise length to depth ratio $(L / D)$ is low, typically $L / D \leq 6$, the shear layer spans across the cavity opening and reattaches on the downstream wall, forming an 'open' cavity flow. ${ }^{1}$ The known fluid dynamic instability processes for open cavities, as reviewed by Rowley \& Williams, ${ }^{2}$ are the Rossiter mechanism, whereby a downstream travelling shearlayer convective instability couples with an upstream feed-back wave inside the cavity, and the wake mode, which is a purely hydrodynamic instability ${ }^{2}$ with global instability type features. ${ }^{3}$

For a given inflow condition and geometry, a cavity fluid dynamic instability can manifest itself as limit cycle modes spanning a specific frequency range. The presence of acoustic or aeroelastic resonant modes in an open cavity over the same frequency range can play an important role in mode selection ${ }^{2}$

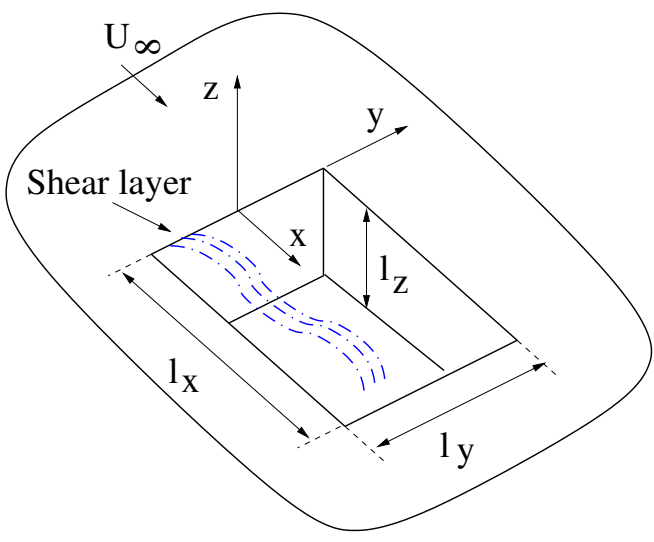

Figure 1. Rectangular cavity. and in phase-locking the feed-back process.

This paper presents a simple analytical method to determine the first six acoustic modes of a rectangular and of a cylindrical enclosure, so that the fluid dynamic practitioner can test whether, at a given operating condition, the instability is susceptible to acoustic resonance driven enhancement.

${ }^{*}$ Lecturer, MAIAA. 


\section{Analytical method}

The analytical method follows closely the conventional approach to determine the room modes in building acoustics. The acoustic pressure fluctuation inside an enclosure at International Standard Atmosphere ground conditions is small compared to the atmospheric pressure, so it can be regarded as an isentropic perturbation of the cavity time-averaged internal pressure. Inside the enclosure, the acoustic pressure fluctuation at location $\mathbf{x}$ and time $t$ is governed by the wave equation

$$
\left(\frac{1}{a_{c}^{2}} \frac{\partial^{2}}{\partial t^{2}}-\nabla^{2}\right) p(\mathbf{x}, t)=0
$$

where $a$ is the speed of sound and subscript $c$ denotes the flow state inside the enclosure. ${ }^{4}$ For a statistically stationary flow, the pressure perturbation can be decomposed into a summation of orthogonal Fourier components

$$
p(\mathbf{x}, t)=\sum_{h=-\infty}^{\infty} p_{h}(\mathbf{x}) \cos \left(\omega_{h} t-\phi_{h}\right)
$$

where $\omega_{h}$ is the $h^{t h}$ Fourier component angular frequency and $\phi_{h}$ is its phase angle at $t=0$. Substituting Eq. (2) in Eq. (1) and imposing that each Fourier component satisfies Eq. (1) independently at all times gives the Helmholtz equation

$$
\left(\nabla^{2}+k_{h}^{2}\right) p_{h}(\mathbf{x})=0
$$

where $k_{h}=\omega_{h} / a_{c}$ is the wavenumber of the $h^{\text {th }}$ Fourier component.

Equation (3) is a second-order partial differential equation, therefore its solution requires imposing two independent boundary conditions per spatial dimension. For an enclosure with rigid solid walls, the wallnormal flow velocity is zero. Therefore, by the conservation of momentum for inviscid flows,

$$
\nabla p_{h}(\mathbf{x}) \cdot \mathbf{n}=0
$$

at the rigid walls, where $\mathbf{n}$ is the inward wall-normal unit vector.

At the cavity open end, a simple acoustic reflecting boundary condition is imposed. This condition is derived from classical duct acoustics, by considering an ideal abrupt expansion, as shown in figure 2. Consider the normal (vertical) flow across the cavity opening induced by the flow-normal (vertical) acoustic velocity just below the $z=0$ plane. A real flow would separate at the cavity mouth, forming a jet that dissipates the kinetic energy of the acoustic velocity by viscous stresses in the jet shear layer. This dissipative mechanism results in a damped oscillation and this process is used, for instance, to design tuned acoustic dampers in a jet engine tail-cone. As this study seeks to identify the cavity undamped natural modes, a flow condition is sought at the $z=0$ plane that

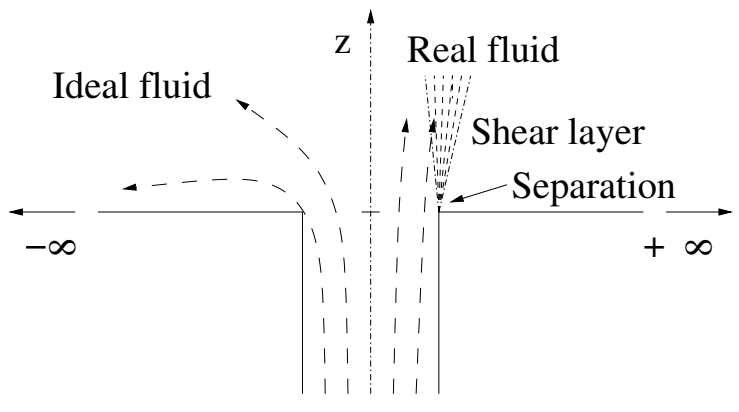

Figure 2. Abrupt flow expansion from a rectangular channel. prevents the onset of this dissipative mechanism. A simple condition that satisfies this requirement is to impose the flow-normal acoustic velocity at the cavity open end to be zero. At this condition, applying the conservation of momentum for inviscid flows in the flow-normal direction gives

$$
\frac{\partial}{\partial z} p_{h}(x, y, 0)=0
$$

A more sophisticated boundary condition for the cavity open end is available in the literature, ${ }^{4}$ derived by considering the dynamic equilibrium of a two-dimensional vortex sheet that models the open cavity shear layer. The use of such a condition would be a useful extension to the present work.

For an arbitrary shaped flat-top open cavity geometry, Eq. (3) can be solved together with the boundary conditions of Eqs. (4) and (5) to determine the acoustic pressure spatial distribution inside the enclosure. 


\section{The natural acoustic modes of a rectangular cavity}

\section{A. The eigen-frequencies}

In the Cartesian reference system of figure 1, Eq. (3) becomes

$$
\left(\frac{\partial^{2}}{\partial x^{2}}+\frac{\partial^{2}}{\partial y^{2}}+\frac{\partial^{2}}{\partial z^{2}}+k_{h}^{2}\right) p_{h}(x, y, z)=0
$$

Similarly, the boundary conditions of Eqs. (4) and (5) become

$$
\begin{aligned}
& \frac{\partial}{\partial x} p_{h}(0, y, z)=0 \\
& \frac{\partial}{\partial x} p_{h}\left(l_{x}, y, z\right)=0 \\
& \frac{\partial}{\partial y} p_{h}\left(x, \pm \frac{l_{y}}{2}, z\right)=0 \\
& \frac{\partial}{\partial z} p_{h}\left(x, y,-l_{z}\right)=0 \\
& \frac{\partial}{\partial z} p_{h}(x, y, 0)=0
\end{aligned}
$$

Equation (6) is solved by the separation of variables approach. Let $p_{h}(x, y, z)=p_{x}(x) p_{y}(y) p_{z}(z)$. Substituting for $p_{h}$ in Eq. (6) and dividing throughout by $p_{x} p_{y} p_{z}$ gives

$$
\frac{1}{p_{x}} \frac{\partial^{2}}{\partial x^{2}} p_{x}+\frac{1}{p_{z}} \frac{\partial^{2}}{\partial z^{2}} p_{z}+k_{h}^{2}=-\frac{1}{p_{y}} \frac{\partial^{2}}{\partial y^{2}} p_{y}
$$

The left hand side of Eq. (12) is independent from $y$ while the right hand side is independent from $x$ and $z$. Therefore, the two sides of the equation must match by a constant $\eta^{2}$, independent from $x, y$ and $z$. This decouples the system into

$$
\begin{aligned}
\frac{1}{p_{x}} \frac{\partial^{2}}{\partial x^{2}} p_{x}+k_{h}^{2}-\eta^{2} & =-\frac{1}{p_{z}} \frac{\partial^{2}}{\partial z^{2}} p_{z} \\
\left(\frac{\partial^{2}}{\partial y^{2}}+\eta^{2}\right) p_{y} & =0
\end{aligned}
$$

Applying recursively the same argument to Eq. (13) by the use of a second constant $\zeta^{2}$ gives

$$
\begin{aligned}
\left(\frac{\partial^{2}}{\partial x^{2}}+k_{h}^{2}-\eta^{2}-\zeta^{2}\right) p_{x} & =0 \\
\left(\frac{\partial^{2}}{\partial y^{2}}+\eta^{2}\right) p_{y} & =0 \\
\left(\frac{\partial^{2}}{\partial z^{2}}+\zeta^{2}\right) p_{z} & =0
\end{aligned}
$$

The general solution of Eq. (17) is $p_{z}=A_{z} \cos \left(\zeta z-\alpha_{\zeta}\right)$, where $A_{z}$ is the amplitude of the pressure fluctuation, independent from $z$, and $\alpha_{\zeta}$ a phase angle to be evaluated using the boundary condition of Eq. (11). Substituting $p_{z}=A_{z} \cos \left(\zeta z-\alpha_{\zeta}\right)$ in Eq. (11) gives $-A_{z} \zeta \sin \left(-\alpha_{\zeta}\right)=0$, which has roots $\zeta=0$, implying a uniform pressure in $z$, and $-\alpha_{\zeta}= \pm n \pi$, with $n$ integer. Therefore, under the boundary condition of Eq. (11), Eq. (17) reduces to $p_{z}=A_{z} \cos (\zeta z \mp n \pi)$. Substituting this in Eq. (10) gives $-A_{z} \zeta \sin \left(-\zeta l_{z} \mp n \pi\right)=0$, which has the roots $\zeta= \pm n \pi / l_{z}$, where $n \geq 0$ is integer. Therefore

$$
p_{z}=A_{z} \cos \left(n \pi z / l_{z}\right)
$$

satisfies Eq. (17) and the boundary conditions of Eqs. (10) and (11). 
Analogously, Eq. (16) has the general solution $p_{y}=A_{y} \cos \left(\eta y-\alpha_{\eta}\right)$. By substituting this in Eq. (9), $\alpha_{\eta}=\mp m \pi / 2$ and $\eta= \pm m \pi / l_{y}$, where $m \geq 0$ is integer. This gives

$$
p_{y}=A_{y} \cos \left(m \pi y / l_{y}-m \pi / 2\right)
$$

that satisfies Eq. (16) with the boundary conditions of Eq. (9).

Let $\xi^{2}=k_{h}^{2}-\eta^{2}-\zeta^{2}$. Substituting for $\xi^{2}$ in Eq. (15), Eq. (15) takes the same form of Eqs. (16) and (17) and has the general solution $p_{x}=A_{x} \cos \left(\xi x-\alpha_{\xi}\right)$. Substituting this in Eqs. (7) and (8) gives $\alpha_{\xi}=0$ and $\xi= \pm l \pi / l_{x}$, where $l \geq 0$ is integer. Therefore,

$$
p_{x}=A_{x} \cos \left(l \pi x / l_{x}\right)
$$

is a solution of Eq. (15) with the boundary conditions of Eqs. (7) and (8).

From the definition of $\xi^{2}$, the cavity natural mode wavenumbers are

$$
k_{l, m, n}^{2}=\xi^{2}+\eta^{2}+\zeta^{2}=\left(\frac{l \pi}{l_{x}}\right)^{2}+\left(\frac{m \pi}{l_{y}}\right)^{2}+\left(\frac{n \pi}{l_{z}}\right)^{2}
$$

and from $\omega_{l, m, n}=a_{c} k_{l, m, n}$, the cavity acoustic eigen-frequencies $f_{l, m, n}=\omega_{l, m, n} /(2 \pi)$ are

$$
f_{l, m, n}=a_{c}\left[\left(\frac{l}{2 l_{x}}\right)^{2}+\left(\frac{m}{2 l_{y}}\right)^{2}+\left(\frac{n}{2 l_{z}}\right)^{2}\right]^{1 / 2}
$$

where $l, m$ and $n$ are arbitrary integers $\geq 0$.

\section{B. The eigenvectors}

The acoustic pressure distribution at the cavity natural frequencies $f_{l, m, n}$ is obtained from combining Eqs. (18), (19) and (20) by $p_{h}(x, y, z)=p_{x}(x) p_{y}(y) p_{z}(z)$. Let $A_{l, m, n}=A_{x} A_{y} A_{z}$, then

$$
p_{l, m, n}(x, y, z)=A_{l, m, n} \cos \left(\pi l x / l_{x}\right) \cos \left(\pi m y / l_{y}-m \pi / 2\right) \cos \left(\pi n z / l_{z}\right)
$$

Figure 3 shows the first six acoustic natural modes of a rectangular cavity of length to depth $L / D=2$ and length to width $L / W=1$. This geometry was investigated numerically by Bissessur et al. ${ }^{5}$ at a free-stream Mach number $M_{\infty}=0.6$. The constant amplitude planes $A_{l, m, n}=-0.5,0$ and 0.5 are shown in figure 3 . The natural modes are shown of unit amplitude and all lengths are normalized by the cavity length $L$. The sequence of figures 3(a)-3(f) shows a standing wave pattern with nodes and anti-nodes, with the number of nodal planes increasing with increasing mode number. The time-dependent acoustic pressure fluctuation inside the enclosure due to acoustic resonance is obtained from the linear combination of all the natural modes in the cavity, according to Eq.(2). As the cavity natural modes are orthogonal, then

$$
p(x, y, z, t)=\sum_{l=0}^{\infty} \sum_{m=0}^{\infty} \sum_{n=0}^{\infty} p_{l, m, n}(x, y, z) \cos \left(2 \pi f_{l, m, n} t-\phi_{l, m, n}\right)
$$

where $\phi_{l, m, n}$ is the phase reference angle of each mode at time $t=0$.

\section{Rectangular cavity instability reinforcement by acoustic resonance}

Equation (22) can be used to diagnose whether, for a given cavity geometry and inflow Mach number, an enclosure is susceptible to acoustic resonance. Consider an enclosure of streamwise length $l_{x}=L$, width $l_{y}=W$ and depth $l_{z}=D$. Multiplying both sides of Eq. (22) by $L / U_{\infty}$, with $U_{\infty}$ being the free-stream velocity of the external flow approaching the cavity, gives the Strouhal numbers of the cavity acoustic modes

$$
\operatorname{Str}_{l, m, n}=\frac{f_{l, m, n} L}{U_{\infty}}=\frac{\left(1+\frac{\gamma-1}{2} M_{\infty}^{2}\right)^{1 / 2}}{M_{\infty}}\left[\left(\frac{l}{2}\right)^{2}+\left(\frac{m}{2} \frac{L}{W}\right)^{2}+\left(\frac{n}{2} \frac{L}{D}\right)^{2}\right]^{1 / 2}
$$

where $\gamma$ is the ratio of the specific heats, $C p / C v$. 


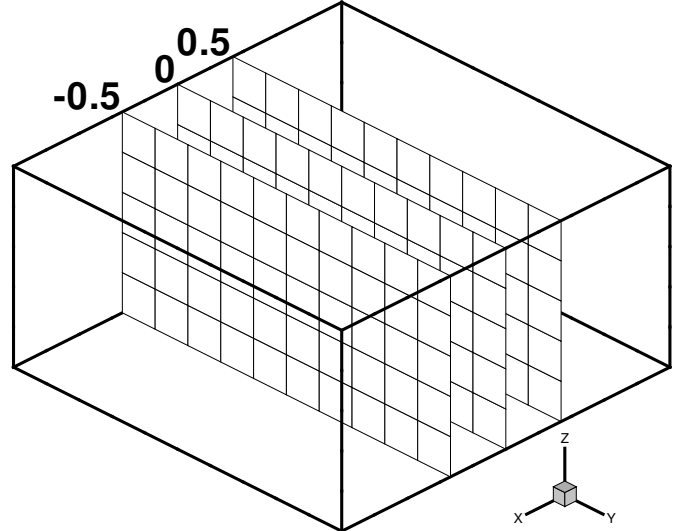

(a) First longitudinal mode

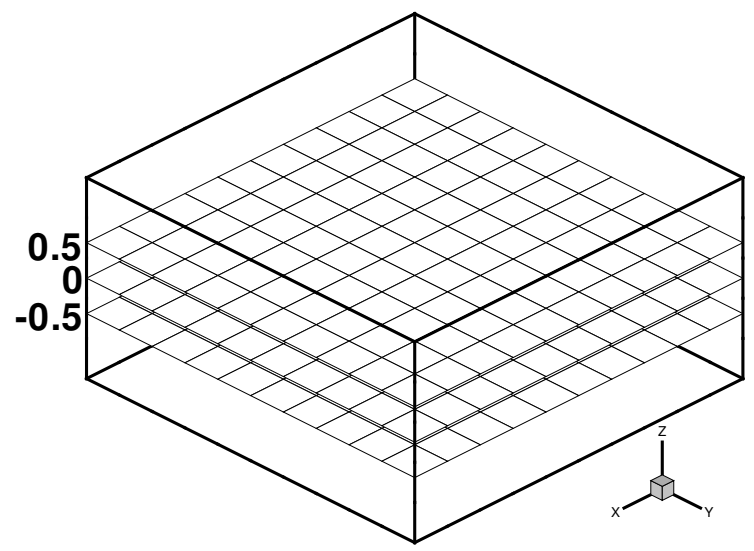

(c) First flow-normal mode

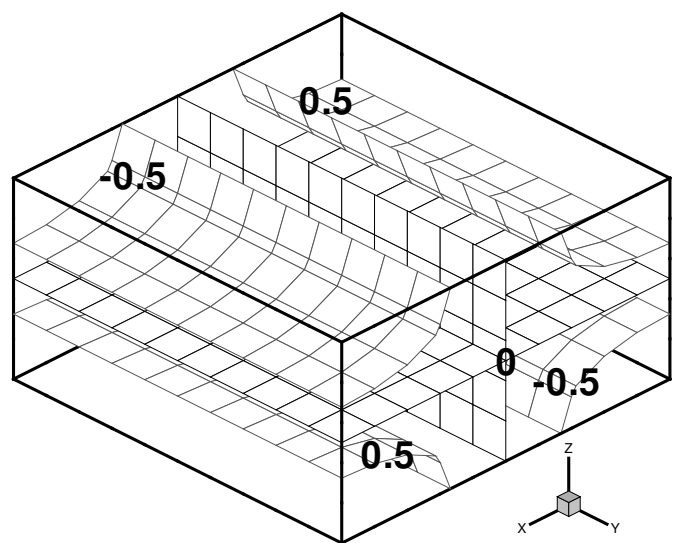

(e) Mode $l=1, m=0, n=1$

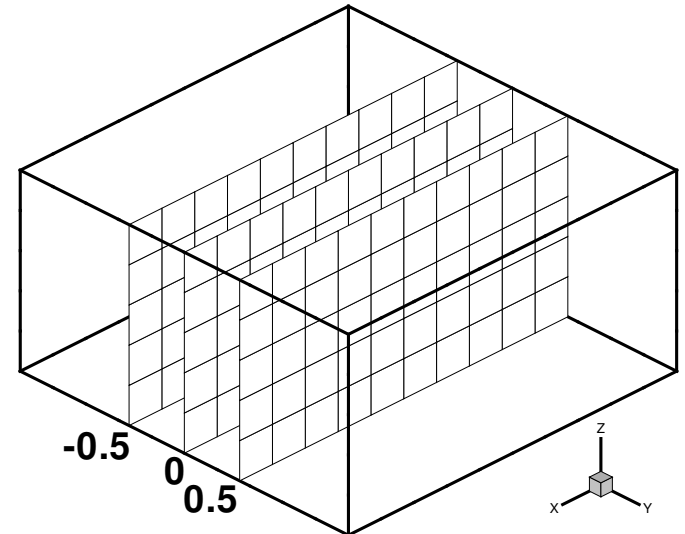

(b) First transverse mode

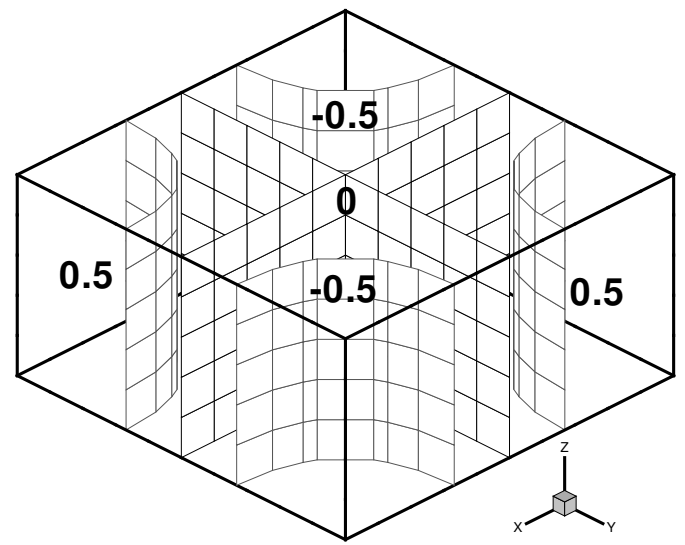

(d) Mode $l=1, m=1, n=0$

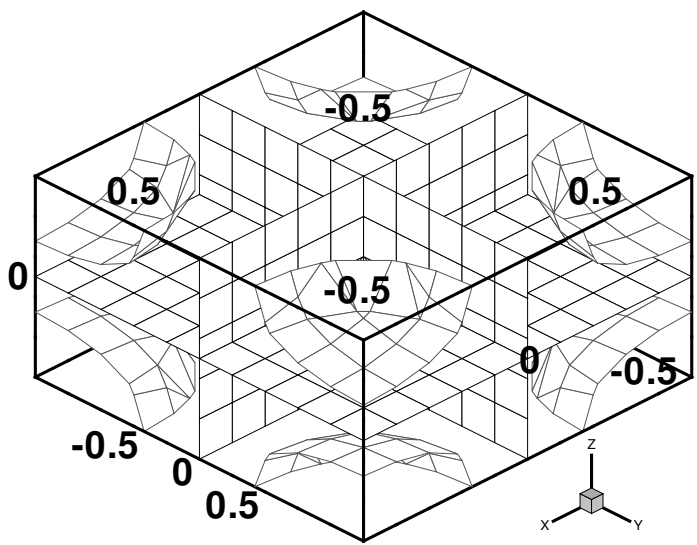

(f) Mode $l=1, m=1, n=1$

Figure 3. Pressure eigenmodes in a rectangular cavity with rigid walls. 
Open cavities that feature a shear layer driven instability, commonly referred to as a Rossiter instability, do so at a Strouhal number that can be predicted by the Rossiter equation, modified by Heller \& Bliss, ${ }^{6}$ which is

$$
\operatorname{Str}_{h}=\frac{h+\alpha}{\frac{M_{\infty}}{\left(1+\frac{\gamma-1}{2} M_{\infty}^{2}\right)^{1 / 2}}+\frac{1}{k_{c}}}
$$

In Eq. (26), $h$ is the Rossiter mode number, which is a positive integer, $\alpha$ is a phase delay in the cavity upstream pressure feed-back, and $k_{c}$ is the average phase speed of shear-layer convective instabilities across the cavity opening, normalized by $U_{\infty}$. Typical values for $\alpha$ and $k_{c}$ are $0.25^{7}$ and 0.57 . $^{7}$

The necessary condition for a Rossiter mode to be reinforced by the cavity acoustic resonance is that the Strouhal number of a cavity acoustic resonance mode matches one component of the harmonic series of any Rossiter mode, that is $\operatorname{Str}_{l, m, n}=o \operatorname{Str}_{h}$, where $o$ is an arbitrary integer. Combining Eq. (25) and Eq. (26), the inflow Mach number at which $\operatorname{Str}_{l, m, n}=o \operatorname{Str}_{h}$ is determined for a given geometry $(L / D, L / W)$. Consider, for instance, the $L / D=2 L / W=1$ rectangular geometry studied by Bissessur et al. ${ }^{5}$ at $M_{\infty}=0.6$. Figure 4 shows the normalized difference in Strouhal numbers, $1-o S t r_{h} / S t_{l, m, n}$, over the Mach number range $0<$ $M_{\infty} \leq 1$. The intercepts of $f\left(M_{\infty}\right)=1-o S t r_{h} / S t r_{l, m, n}$ with the abscissa define the Mach numbers at which the Strouhal numbers match, giving acoustic reinforcement of a Rossiter mode. $f\left(M_{\infty}\right)$ is shown in figure 4 for the manifold $(1 \leq h \leq 2,1 \leq l \leq 2, m=0, n=0,1 \leq o \leq 2)$ that

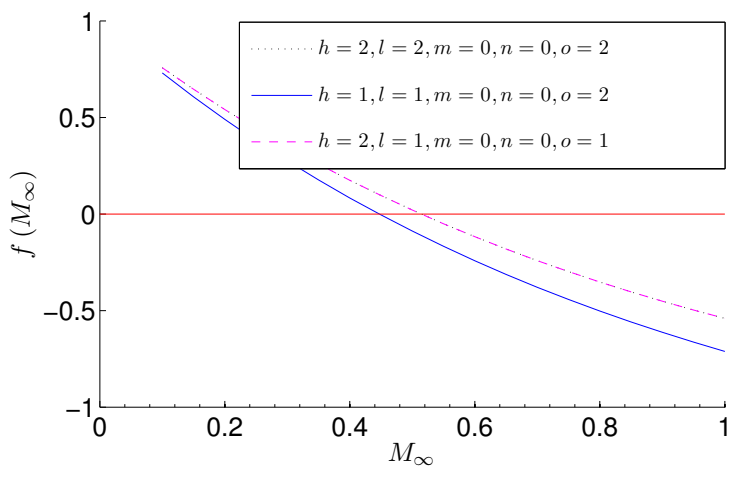

Figure 4. Normalized difference between the Strouhal number for longitudinal acoustic resonance and the Strouhal number of the first two Rossiter modes. was identified by investigating numerically the sixdimensional parameter space as having zeros close to the cavity test conditions of Bissessur et al. ${ }^{5}$ The results indicate that, at the selected test Mach number of 0.6 of Bissessur et al., ${ }^{5}$ there is a $12 \%$ difference between the Strouhal number of the second Rossiter mode and that of the first longitudinal acoustic mode. Likewise, there is a $12 \%$ difference between the Strouhal number of the second harmonic of the second Rossiter mode and that of the second longitudinal acoustic mode (the black dotted curve and the magenta dashed curve overlap). The second Rossiter modes predicted numerically by Bissessur et al. ${ }^{5}$ has a Strouhal number 0.84 , which is $3 \%$ away from the first longitudinal acoustic mode Strouhal number. The proximity of the Strouhal numbers indicate that acoustic resonance reinforcement is likely to have affected the second Rossiter mode at this cavity test Mach number.

\section{The natural acoustic modes of a cylindrical cavity}

\section{A. The eigen-frequencies}

In the cylindrical coordinates of figure 5, the Helmholtz equation, Eq. (3), becomes

$$
\left(\frac{\partial^{2}}{\partial r^{2}}+\frac{1}{r} \frac{\partial}{\partial r}+\frac{1}{r^{2}} \frac{\partial^{2}}{\partial \theta^{2}}+\frac{\partial^{2}}{\partial z^{2}}+k_{h}^{2}\right) p_{h}(r, \theta, z)=0
$$

where subscript $h$ indicates the $h^{\text {th }}$ Fourier component of the time-dependent acoustic pressure fluctuation $p(r, \theta, z, t)$, as defined in Eq. (2). The boundary conditions of Eqs. (4) and (5) are re-cast in cylindrical coordinates as

$$
\begin{aligned}
p_{h}(r, \theta, z) & =p_{h}(r, \theta+2 \pi, z) \\
p_{h}(0, \theta, z) & \in \Re \\
\left(\frac{\partial}{\partial r}+\frac{1}{r}\right) p_{h}(R, \theta, z) & =0 \\
\frac{\partial}{\partial z} p_{h}(r, \theta,-D) & =0
\end{aligned}
$$




$$
\frac{\partial}{\partial z} p_{h}(r, \theta, 0)=0
$$

Whereas there is a straight-forward correspondence between Eq. (5) and Eq. (32), the relationship between Eq. (4) and Eqs. (28)-(31) is less immediate. Recall that, by virtue of the Helmholtz equation being a second-order partial differential equation, its integration requires exactly two independent boundary conditions per spatial dimension. As there is no solid boundary normal to $\theta$ in figure 5 , Eq. (4) is always satisfied in the $\theta$ direction by virtue of the $\theta$-normal boundaries having zero extent. Therefore two replacement boundary conditions are required in $\theta$ to integrate Eq. (27). These are obtained by imposing that the pressure field in $\theta$ matches itself with a periodicity of $2 \pi$, as stated in Eq. (28). It is worth noting that Eq. (28) constitutes two boundary conditions in $\theta$, imposed respectively on the $\theta=0$ lower $\theta$ boundary and on the $\theta=2 \pi$ upper $\theta$ boundary.

In the radial direction, it is imposed that the acoustic pressure fluctuation at the origin is finite, which gives Eq. (29), and that the acoustic particle

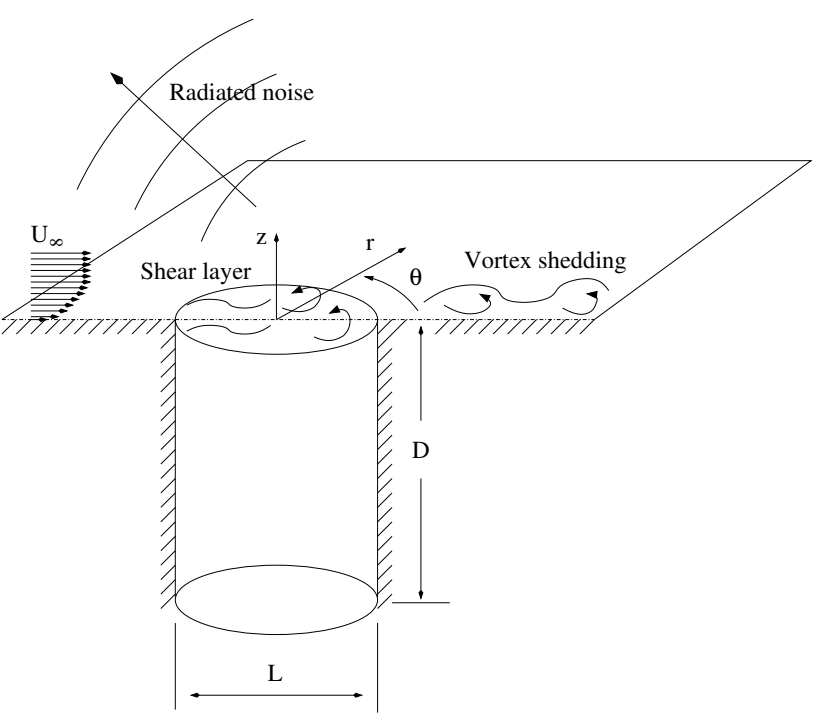

Figure 5. Cylindrical cavity geometry. velocity normal to the cylindrical solid wall of diameter $L=2 R$ is zero, which is Eq. (4) applied on the cylindrical wall that gives Eq. (30). Finally, applying Eq. (4) on the cavity solid floor gives Eq. (31) that completes the set of boundary conditions for a cylindrical open cavity.

The solution of Eq. (27) with the boundary conditions of Eqs. (28)-(32) follows the same separation of variables approach as for the Helmholtz equation in Cartesian coordinates, detailed in Subsection III.A. Let $p_{h}(r, \theta, z)=p_{r}(r) p_{\theta}(\theta) p_{z}(z)$, then Eq. (27) decouples into

$$
\begin{aligned}
\left(\frac{\partial^{2}}{\partial r^{2}}+\frac{1}{r} \frac{\partial}{\partial r}+k_{h}^{2}-\frac{\eta^{2}}{r^{2}}-\zeta^{2}\right) p_{r} & =0 \\
\left(\frac{\partial^{2}}{\partial \theta^{2}}+\eta^{2}\right) p_{\theta} & =0 \\
\left(\frac{\partial^{2}}{\partial z^{2}}+\zeta^{2}\right) p_{z} & =0
\end{aligned}
$$

where $\eta$ and $\zeta$ are constants, independent from $r, \theta$ and $z$.

Equation (35) with the boundary conditions of Eqs. (31) and (32) is identical to its Cartesian counterpart, Eq. (17) with the boundary conditions of Eqs. (10) and (11). Therefore Eq. (18) with $l_{z}=D$ satisfies Eq. (35) and the boundary conditions of Eqs. (31) and (32).

Equation (34) has the same form of its Cartesian counterpart, Eq. (16), so its general solution is $p_{\theta}=$ $A_{\theta} \cos \left(\eta \theta-\alpha_{\eta}\right)$. Substituting this in Eq. (28) gives $\eta= \pm m$, where $m \geq 0$ is an integer. Therefore

$$
p_{\theta}=A_{\theta} \cos \left( \pm m \theta-\alpha_{m}\right)
$$

where $\alpha_{m}$ is the phase-shift angle with respect to the $\theta=0$ direction. $\alpha_{m}$ remains indeterminate, as there is no solid boundary nor other reference to lock any of the azimuthal modes to a specific phase angle in a cylindrical cavity with smooth walls. The numerical experiments by Grottadaurea \& Rona ${ }^{8,9}$ indicate that $\alpha_{m}$ tends to align normal to the azimuthal direction of the free-stream flow velocity above the cavity opening.

In Eq. (36), $\eta=+m$ and $\eta=-m$ satisfy independently the boundary conditions of Eq. (28), therefore the azimuthal acoustic pressure fluctuation can be made up of any arbitrary combination of anti-clockwise $+m$ modes and clockwise $-m$ modes of arbitrary amplitudes $A_{\theta}$ and $B_{\theta}$ and phase angles $\alpha_{m}$ and $\beta_{m}$. This allows to re-cast Eq. (36) as

$$
p_{\theta}=A_{\theta} \cos \left(m \theta-\alpha_{m}\right)+B_{\theta} \cos \left(-m \theta-\beta_{m}\right)
$$


Let $\xi^{2}=k_{h}^{2}-\zeta^{2}$. Substituting for $\xi$ and for $\eta= \pm m$ in Eq. (33) gives

$$
\left(\frac{\partial^{2}}{\partial r^{2}}+\frac{1}{r} \frac{\partial}{\partial r}+\xi^{2}-\frac{m^{2}}{r^{2}}\right) p_{r}=0
$$

The general solution to Eq. (38) is $p_{r}=A_{r} J_{m}(\xi r)+B_{r} Y_{m}(\xi r)$, where $A_{r}$ and $B_{r}$ are the amplitudes of the radial acoustic pressure fluctuation, independent from $r, J_{m}$ is the $m^{\text {th }}$ order Bessel function of the first kind and $Y_{m}$ is the $m^{\text {th }}$ order Bessel function of the second kind. As $Y_{m}$ is singular at $r=0$, by the boundary condition of Eq. (29), $B_{r}=0$. Substituting $p_{r}=A_{r} J_{m}(\xi r)$ in Eq. (30) gives

$$
\xi r \frac{\partial}{\partial \xi r} J_{m}(\xi R)+J_{m}(\xi R)=0
$$

and using the Bessel function property $\xi r J_{m}^{\prime}(\xi r)=m J_{m}(\xi r)-\xi r J_{m+1}(\xi r)$

$$
\frac{J_{m}(\xi R)}{J_{m+1}(\xi R)}=\frac{\xi R}{m+1}
$$

Equation (40) can be solved numerically for $\xi$, to determine the wavenubers that satisfy the radial component of the Helmholtz equation with a cylindrical rigid wall as boundary condition. Figure 6 shows $f_{m}(\xi R)=(m+1) J_{m}-\xi R J_{m+1}$ plotted over the range $0 \leq \xi R \leq 10$. $f_{m}$ is evaluated for five values of $m$, specifically, $m=0$, representing the axisymmetric modes, $m= \pm 1$ representing the first clockwise and anti-clockwise azimuthal or spinning modes, and $m=2$ and 3, representing higher order anti-clockwise spinning modes. The intercepts of $f_{m}$ with the abscissa are the roots of Eq. (40). Due to the Bessel function property $J_{m}=(-1)^{m} J_{-m}$, $f_{m}=(-1)^{m} f_{-m}$, which implies that clockwise and anti-clockwise spinning modes of the same order $|m|$ share the same eigen-value root of Eq. (40), therefore they have the same propagation characteristic.

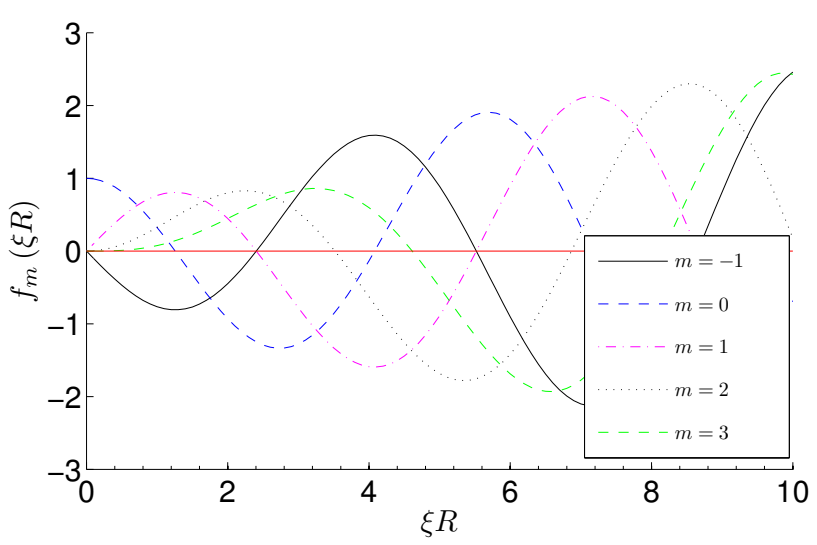

Figure 6. Eigenfrequency function.

This enables an easy energy transfer between these mode pairs, so that, if a cylindrical cavity is forced at one spinning mode eigen-frequency, azimuthal waves may appear with a dominant clockwise or anti-clockwise component. When the clockwise and anti-clockwise mode pair have about the same modal amplitude, the instability will appear as an antisymmetric fluctuation about the $\theta=\alpha_{m}+\pi / 2$ axis. An antisymmetric instability is predicted by a numerical cylindrical cavity model of Grottadaurea \& Rona, ${ }^{8,9}$ although the frequency predicted by the numerical model is lower, indicating that the antisymmetric instability is probably fluid-dynamic rather than due to resonant acoustic modes.

The oscillatory nature of the Bessel function is such that, for any given $m$, there is an infinite number of intersections of $f_{m}$ with the abscissa over the range $\xi R \geq 0$. This gives three intersections or roots of Eq. (40) in the range $0 \leq \xi R \leq 10$ for $m=0$ and similar multiple roots for $m \neq 0$. Table 1 lists the first three intersections along the $\xi R$ axis in the positive direction, starting from $\xi R=0$. These have been numbered consecutively for increasing $\xi R$, so that $\xi_{l, m} R$ is the $l^{t h}$ root of the azimuthal mode $m$, where $l \geq 0$ is integer. The radial

Table 1. Cylindrical cavity natural circular wavenumbers, $\xi_{l, m} R$.

\begin{tabular}{|c|c|c|c|c|}
\hline$m$ & 0 & \pm 1 & 2 & 3 \\
\hline \hline$l=0$ & - & 0 & 0 & 0 \\
\hline$l=1$ & 1.2556 & 2.4048 & 3.5180 & 4.6123 \\
\hline$l=2$ & 4.0793 & 5.5201 & 6.8661 & 8.1576 \\
\hline
\end{tabular}
acoustic pressure distribution is therefore given by

$$
p_{r}=A_{l, m} J_{m}\left(\xi_{l, m} r\right)
$$

The roots in table 1 were obtained by evaluating $f_{m}$ over the range $0 \leq \xi R \leq 10$ at increments of $\Delta \xi R=0.1$ and by interpolating linearly between each $\xi R$ pair lying on either side of each zero crossing. 
This technique was selected in favour of a conventional Newton-Raphson iteration to prevent root skipping, due to the oscillatory nature of the Bessel function. The results in table 1 on the preceding page are therefore accurate to two decimal places. As $f_{m}$ is monotonic over the interval \pm 0.005 about each stated root, a Newton-Raphson iteration can make use of the values in table 1 on the page before as the starting point to reach an arbitrarily high level of significant digits.

From table 1 on the preceding page it can be noticed that the roots are interlaced, so that $\xi_{l, m} R<$ $\xi_{l, m+1} R<\xi_{l+1, m} R$. This indicates that a higher azimuthal mode intercalates two consecutive radial modes in frequency. The $m=0$ axisymmetric modes can be shown to give rise to an acoustic particle velocity distribution that is singular at $r=0$. This arises from the conservation of momentum of cylindrical waves collapsing on the axis of symmetry. Such a singularity is non-physical and would be prevented by the action of viscosity in a real fluid, which would dissipate the modal energy into heat. Therefore, the cylindrical cavity cannot support an undamped standing axisymmetric wave and the axisymmetric modes can only occur as damped modes in the system. The reader should be mindful of such a feature for the purpose of establishing the lowest self-sustained acoustic resonant eigenvalue of a cylindrical cavity.

From $\xi^{2}=k_{h}^{2}-\zeta^{2}, \omega_{h}=a_{c} k_{h}$ and $\omega_{h}=2 \pi f_{h}$, then the acoustic resonant eigenvalues for a cylindrical cavity are

$$
f_{l, m, n}=\frac{a_{c}}{2 \pi}\left[\xi_{l, m}^{2}+\left(\frac{n \pi}{D}\right)^{2}\right]^{1 / 2}
$$

where $l, m$ and $n$ are integers $\geq 0$ and $\xi_{l, m}$ is obtained from table 1 by dividing the stated values by the cylinder radius $R$.

\section{B. The eigenvectors}

The acoustic pressure perturbation at any given acoustic resonance frequency $f_{l, m, n}$ inside a cylindrical cavity is obtained by combining Eqs. (18), (37) and (41) by $p_{h}(r, \theta, z)=p_{r}(r) p_{\theta}(\theta) p_{z}(z)$. This gives

$p_{l, m, n}(r, \theta, z)=A_{l, m, n} J_{m}\left(\xi_{l, m} r\right) \cos \left(m \theta-\alpha_{m}\right) \cos (n \pi z / D)+B_{l, m, n} J_{m}\left(\xi_{l, m} r\right) \cos \left(-m \theta-\beta_{m}\right) \cos (n \pi z / D)$

Given that $J_{-m}=(-1)^{m} J_{m}$, by factorizing $(-1)^{m}$ out of the mode amplitude $B_{l, m, n}$, Eq.(43) can be re-cast as

$$
p_{l, m, n}(r, \theta, z)=A_{l, m, n} J_{m}\left(\xi_{l, m} r\right) \cos \left(m \theta-\alpha_{m}\right) \cos (n \pi z / D)
$$

where $0 \leq l<\infty,-\infty<m<\infty$ and $0 \leq n<\infty$ are arbitrary integers.

Figures 7 and 8 show the first six acoustic resonant spinning modes of a cylindrical cavity of diameter to depth ratio $L / D=0.714$. This is the geometry investigated numerically by Grottadaurea \& Rona. ${ }^{8,9}$ The natural modes are shown of unit amplitude on the $z=-D / 2$ plane, lengths are normalized by the cavity diameter $L$ and the phase reference angle $\alpha_{m}=\pi / 2$ for all $m$. There is some similarity between the second spinning mode $(l, m, n)=(1,2,0)$ and the anti-symmetric shear-layer pattern predicted by Grottadaurea \& Rona $^{8,9}$ across the cavity opening.

\section{Cylindrical cavity instability reinforcement by acoustic resonance}

Equation (42) can be used to determine whether, for a given cylindrical cavity diameter to depth ratio, a fluid dynamic instability is likely to be reinforced by acoustic resonance. Let $L$ be the cavity diameter and $U_{\infty}$ the free-stream velocity of a flow blowing tangentially over the cavity opening. The cylindrical cavity acoustic natural frequencies can be re-stated as Strouhal numbers

$$
\operatorname{Str}_{l, m, n}=\frac{f_{l, m, n} L}{U_{\infty}}=\frac{\left(1+\frac{\gamma-1}{2} M_{\infty}^{2}\right)^{1 / 2}}{M_{\infty}}\left[\left(\frac{\xi_{l, m} R}{\pi}\right)^{2}+\left(\frac{n}{2} \frac{L}{D}\right)^{2}\right]^{1 / 2}
$$

At an inflow Mach number of 0.3 , numerical predictions indicate that a $L / D=0.714$ cavity exhibits a fluid dynamic self-sustained instability at $S t r=0.491 .^{8,9}$ From Eq. (45), the closest Strouhal number for acoustic resonance is found to be 2.57, indicating that the two instability mechanisms are not interacting at the selected flow conditions. At the reduced inflow Mach number of 0.235 , the predicted fluid dynamic Strouhal number is 0.5295 . The closest Strouhal number for acoustic resonance is $S t r=3.27$, so, at this reduced Mach number, coupling between the two instabilities is likely not to have occurred. 


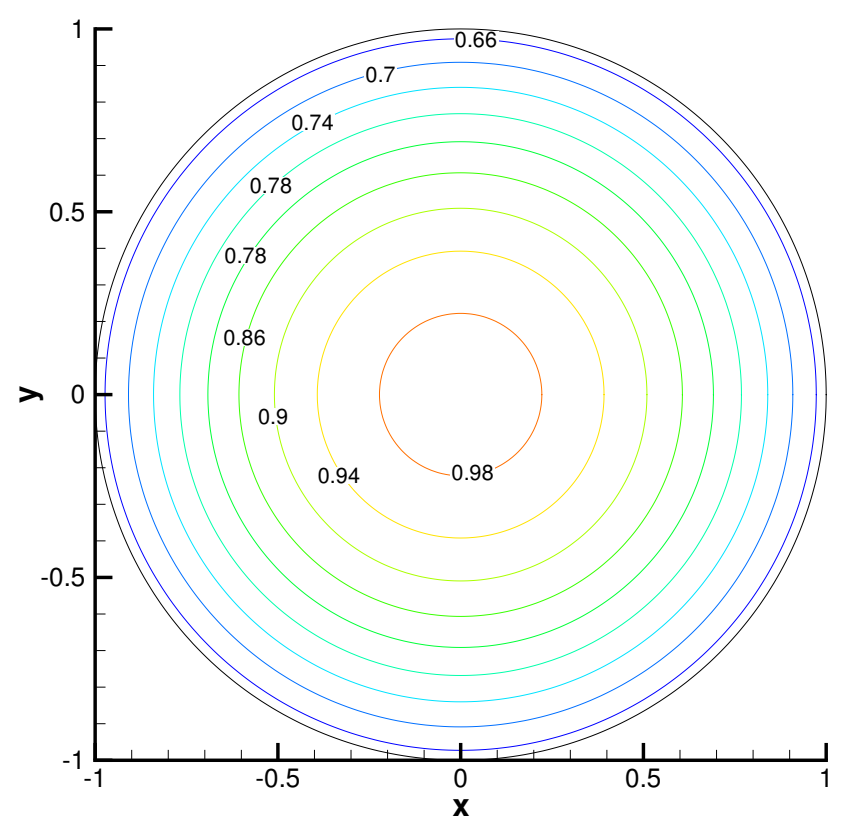

(a) First axisymmetric mode, $l=1, m=0, n=0$

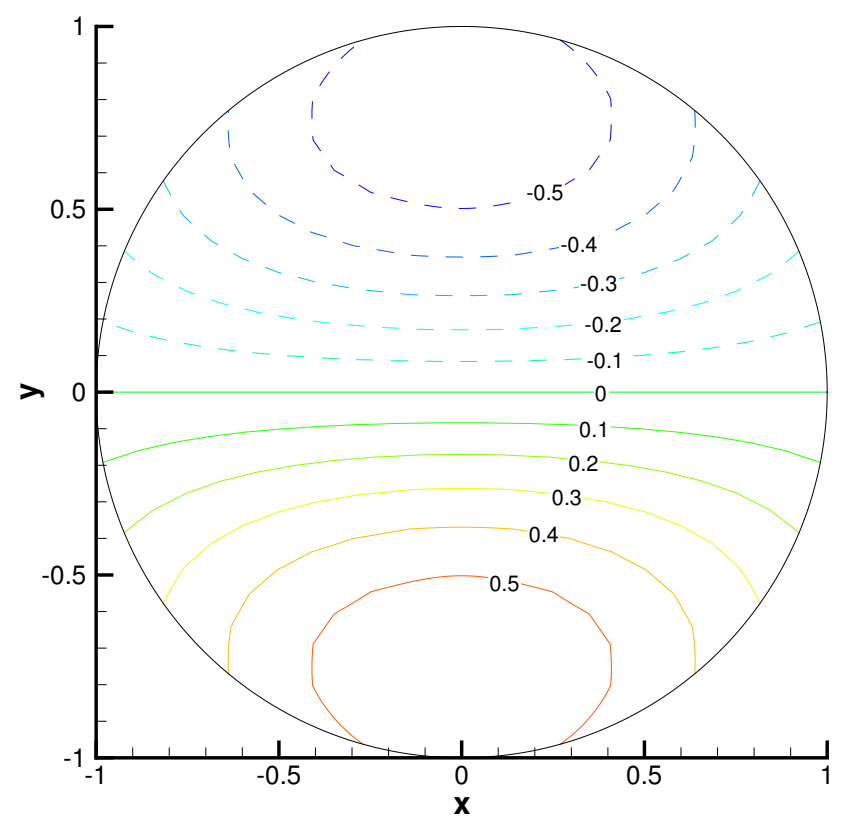

(c) First spinning mode, $l=1, m=1, n=0$

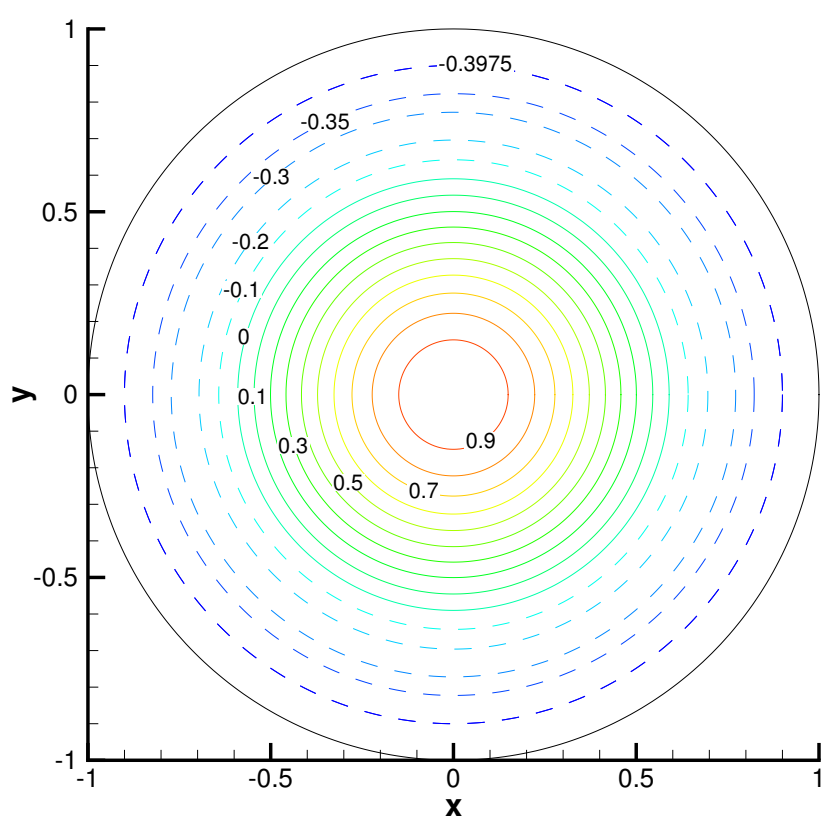

(b) Second axisymmetric mode, $l=2, m=0, n=0$

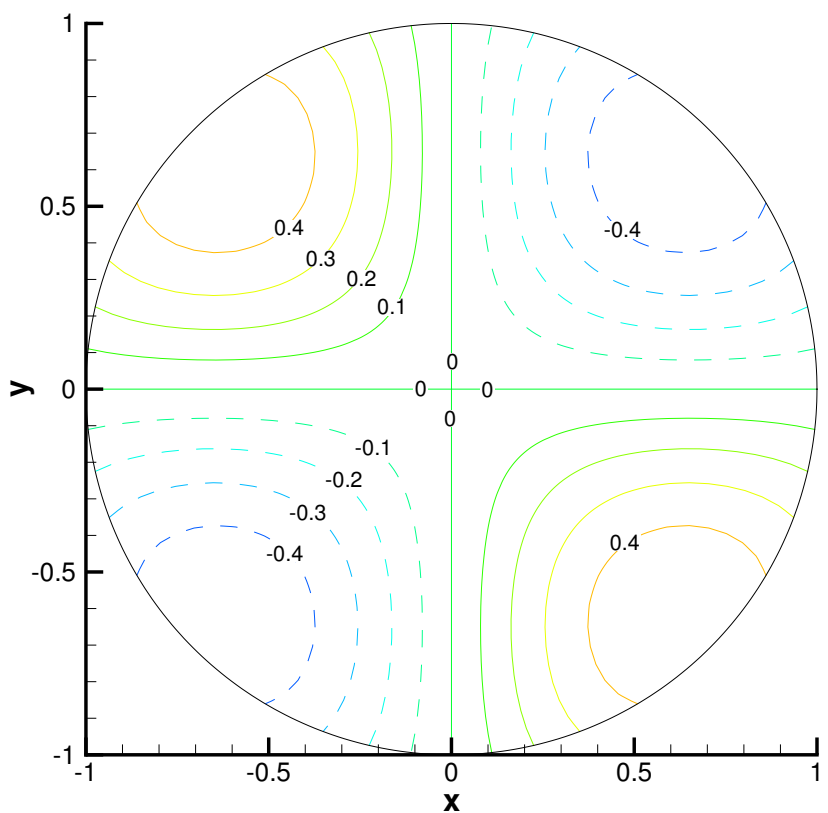

(d) Second spinning mode, $l=1, m=2, n=0$

Figure 7. Pressure eigenmodes in a cylinder with rigid walls. 


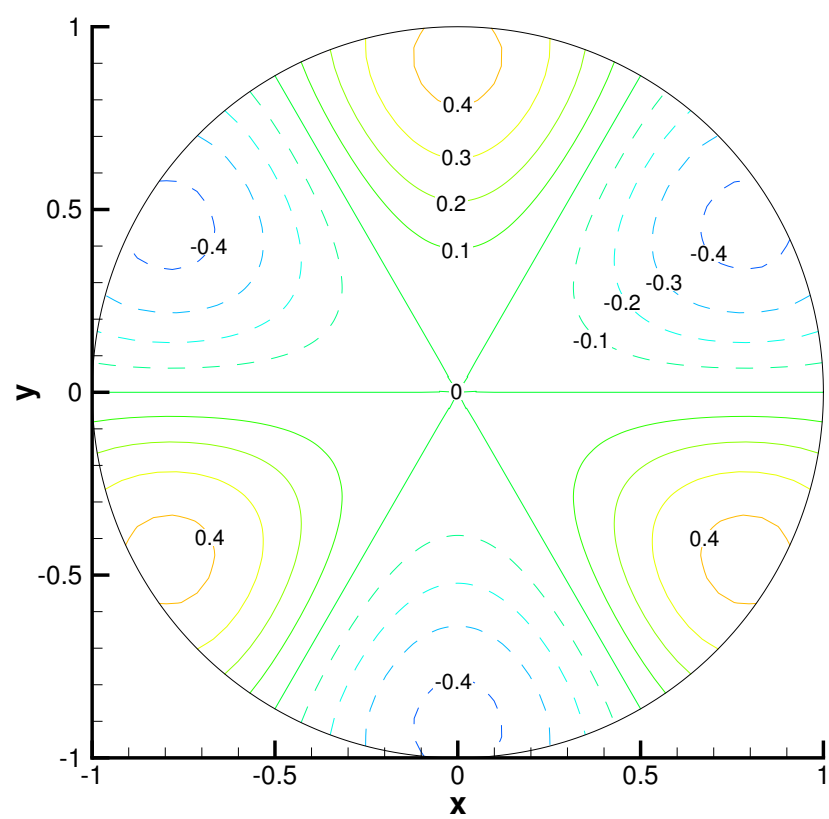

(a) Third spinning mode, $l=1, m=3, n=0$

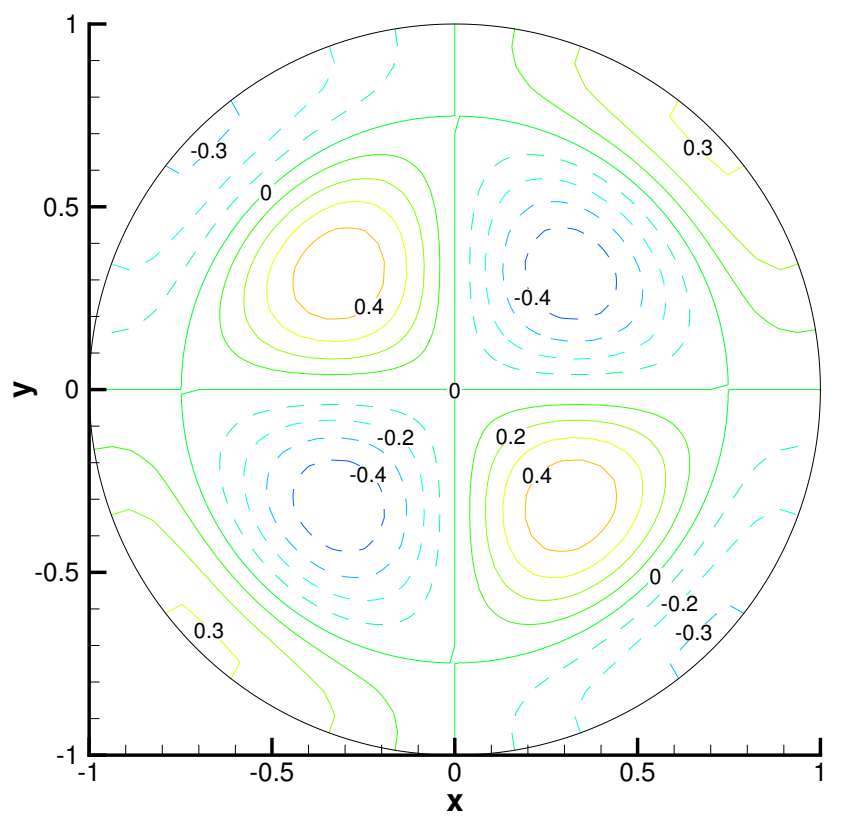

(c) Fifth spinning mode, $l=2, m=2, n=0$

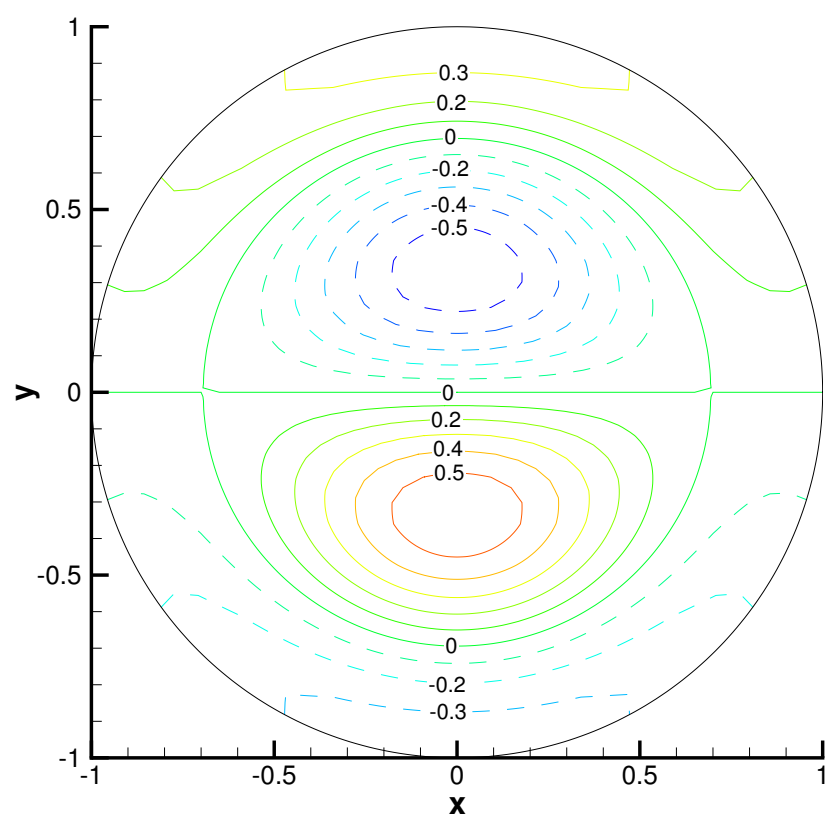

(b) Fourth spinning mode, $l=2, m=1, n=0$

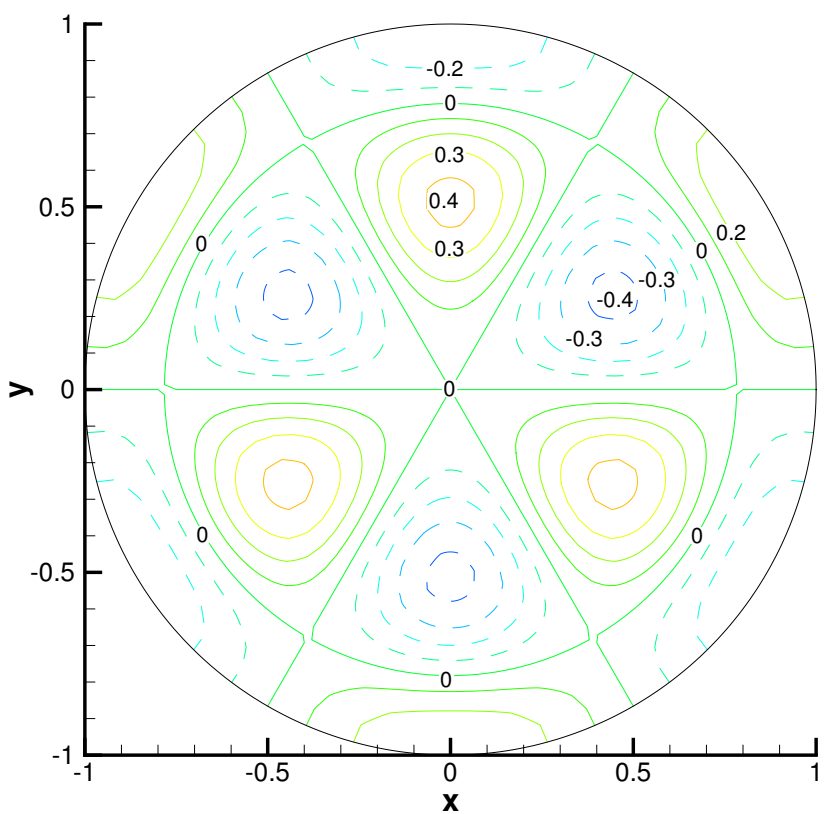

(d) Sixth spinning mode, $l=2, m=3, n=0$

Figure 8. Pressure eigenmodes in a cylinder with rigid walls. 
At the same free-stream Mach numbers of 0.3 and 0.235 , a $L / D=2.5$ cylindrical cavity exhibits a fluid dynamic instability at $S t r=1.448$ and $S t r=0.833$, respectively. The corresponding closest acoustic resonance modes are $S t r=2.57$ and $S t r=3.27$, showing that coupling between the two instabilities did not occur.

\section{Conclusion}

An analytical method is presented to estimate the acoustic resonant modes of an open cavity, which is applicable to an arbitrary shaped enclosure. The method is applied to determine the acoustic resonant eigen-frequencies and mode shapes of a rectangular and of a cylindrical cavity.

The model is of practical use to determine whether acoustic resonance can reinforce fluid dynamic instabilities that often characterize open cavity flows. As a practical example, the interaction between the acoustic resonance and the Rossiter modes in an $L / D=2, L / W=1$, Mach 0.6 model cavity flow was considered. It was found that, at the selected test conditions, the coupling between the fluid dynamic and acoustic instabilities was likely for the Rossiter 2 mode and its second harmonic.

The application of the analytical method to a cylindrical geometry highlighted salient physical aspect of acoustic resonance in a cylinder with rigid walls. It was determined that an axisymmetric standing wave pattern cannot be realized in a real flow, due to the high acoustic particle velocity that occurs at the cylinder axis. The absence of a geometrical reference in the azimuthal direction around smooth cavity walls leaves the zero phase angle of azimuthal modes indeterminate. In practice, this means that azimuthal cavity resonant modes may precess over time. Therefore, a control strategy for such a flow resonant instability may start by considering the addition of a tab around the walls to act as a phase reference lock for the azimuthal modes, so to reduce the degrees of freedom the controller has to tackle. Also, when experiments are conducted on a cylindrical cavity flow, attention must be paid to any azimuthal precession of any nodal plane of the flow instabilities during the test, for instance, by including an adequate monitoring strategy of the pressure phase around the cylinder perimeter.

Th analytical method was used to check for any frequency coupling between the aerodynamic instability of a cylindrical cavity with aspect ratios 0.714 and 2.5 tested at Mach numbers 0.235 and 0.3 and the acoustic modes of the cylinder. No evidence of such coupling was found over the range of acoustic mode numbers tested.

\section{Acknowledgement}

This research project has been supported by a Marie Curie Early Stage Research Training Fellowship of the European Community's Sixth Framework Programme under contract number MEST CT 2005020301. The help of Marco Cambiano in the initial stage of the research is acknowledged.

\section{References}

${ }^{1}$ Charwat, A., Roos, J., Dewey, F., and Hitz, J., "An investigation of separated flows. Part I: The pressure field," J. Aerospace Sci., Vol. 28, No. 6, 1961, pp. 457-70.

${ }^{2}$ Rowley, C. and Williams, D., "Dynamics and control of high-Reynolds-number flow over open cavities," Annual Review of Fluid Mechanics, Vol. 38, 2006, pp. 251-276.

${ }^{3}$ Theofilis, V., "Advances in global linear instability analysis of nonparallel and three-dimensional flows," Progress in Aerospace Sciences, Vol. 39, 2003, pp. 249-315.

${ }^{4}$ Covert, E., "An approximate calculation of the onset velocity of cavity oscillations," Journal of the American Institute of Aeronautics and Astronautics, Vol. 8, No. 12, Dec. 1970, pp. 2189-2194.

${ }^{5}$ Bissessur, R., Chen, X., and Zhang, X., "Numerical investigation of subsonic 2D/3D cavity flows," Conference Paper 2004-0683, AIAA, 2004, 42nd AIAA Aerosopace Sciences Meeting and Exhibit, 5-8 January 2004, Reno, NV.

${ }^{6}$ Heller, H. and Bliss, D., "The physical mechanism of flow induced pressure fluctuations in cavities and concepts for their suppression," Conference Paper 75-91, AIAA, 1975.

${ }^{7}$ Tam, C. and Block, P., "On the tones and pressure oscillations induced by flow over rectangular cavities," J. Fluid Mechanics, Vol. 89, 1978, pp. 373-399.

${ }^{8}$ Grottadaurea, M. and Rona, A., "Noise sources from a cylindrical cavity," Conference Paper 2007-3723, 13th AIAA/CEAS Aeroacoustics Conference, Rome, Italy, 2007.

${ }^{9}$ Grottadaurea, M. and Rona, A., "The role of the inflow momentum thickness in subsonic cylindrical cavity noise generation," 14th International Congress on Sound and Vibration, 2007. 